\title{
Optimized Anisotropic Rotational Invariant Diffusion Scheme on Cone-Beam CT
}

\author{
Dirk-Jan Kroon ${ }^{1}$, Cornelis H. Slump ${ }^{1}$, and Thomas J.J. Maal ${ }^{2}$ \\ ${ }^{1}$ Signals and System, University of Twente, The Netherlands \\ ${ }^{2}$ Radboud University Nijmegen Medical Center, The Netherlands
}

\begin{abstract}
Cone-beam computed tomography (CBCT) is an important image modality for dental surgery planning, with high resolution images at a relative low radiation dose. In these scans the mandibular canal is hardly visible, this is a problem for implant surgery planning. We use anisotropic diffusion filtering to remove noise and enhance the mandibular canal in CBCT scans. For the diffusion tensor we use hybrid diffusion with a continuous switch (HDCS), suitable for filtering both tubular as planar image structures. We focus in this paper on the diffusion discretization schemes. The standard scheme shows good isotropic filtering behavior but is not rotational invariant, the diffusion scheme of Weickert is rotational invariant but suffers from checkerboard artifacts. We introduce a new scheme, in which we numerically optimize the image derivatives. This scheme is rotational invariant and shows good isotropic filtering properties on both synthetic as real CBCT data.
\end{abstract}

\section{Introduction}

Cone-beam computed tomography (CBCT) is an increasingly utilized imaging modality for dental surgery planning [1, due to the low hardware cost and high resolution images at a relative low radiation dose. For the surgical planning of implants, the mandibular nerve canals have to be segmented. In these scans the mandibular nerve canals are hardly visible. In implant placement, the segmentation is used to guard the safety margin around the canals during surgery. CBCT scanners have a relatively low radiation dose [1] thus the small mandibular canal is characterized by low contrast in a noisy image, see figure 4. The research goal of this paper, is to find a method to improve image contrast in CBCT scans for small structures.

Currently the best way to improve contrast in a CT image is to apply iterative reconstruction methods with regularization to suppress streak-artifacts and to improve smoothness in uniform regions [2]. In practice CBCT systems do not provide the required raw-scanner data for this approach. Therefore post reconstruction noise filtering is the practical method to improve image quality. A medical image is often assumed to have piecewise smooth regions with oscillatory noise, separated by sharp edges. There are many methods available in the literature to denoise such an image [3], in this paper we focus on edge enhancing diffusion filtering.

T. Jiang et al. (Eds.): MICCAI 2010, Part III, LNCS 6363, pp. 221-228, 2010.

(C) Springer-Verlag Berlin Heidelberg 2010 
Linear diffusion equals Gaussian filtering in which the diffusion time controls the smoothing scale. To preserve the edges Perona-Malik introduced regularized non-linear diffusion (RPM) 4]. Edge preservation is achieved by lowering the scalar diffusion constant in the neighborhood of steep edges. This method results in piecewise smooth regions, however, image edges remain noisy. Instead of using a scalar diffusion constant, a tensor can be used to adapt the diffusion to the underlying image structure. So we smooth with small elongated kernels along edges, and Gaussian like kernels in uniform regions. The tensor can be constructed in two ways, as a coherence-enhancing diffusion (CED) 5 or as an edge-enhancing diffusion (EED). Recently the CED and EED algorithms were combined in an hybrid diffusion filter with a continuous switch (HDCS) 6]. If the local image structure is tubular HDCS switches to CED and if it is planar to EED.

The focus of this paper are the discretization schemes of the anisotropic diffusion tensor. We will evaluate the performance of the standard discretization scheme and the rotational invariant scheme of Weickert [7, and introduce a new scheme in which optimal filtering kernels are constructed using numerical optimization.

This paper is organized as follows, in the second section we introduce the diffusion filtering algorithm and discretization schemes. The new optimized scheme is introduced in the third section. Followed by evaluation of the diffusion schemes on synthetic and real images, and by the final section with discussion and conclusions.

\section{Diffusion Filtering}

Anisotropic diffusion filtering is an iterative edge preserving smoothing method. It describes the local image structure using a structure tensor also referred to as the "second-moment matrix", for details see [5]. This descriptor is transformed into a diffusion tensor $\mathbf{D}$. The diffusion equation is commonly written in an iterative forward difference approximation [7]:

$$
\frac{\partial u}{\partial t}=\nabla \cdot(\mathbf{D} \nabla u) \Rightarrow u_{k+1} \cong u_{k}+(\nabla \cdot(\mathbf{D} \nabla u))
$$

Where $u(u=u(t, x, y, z))$ is the image, $x, y, z$ the pixel coordinates and $t$ the diffusion time. In the discrete function the continues time is replaced by, $\tau$ the time step-size and $k$ the number of the iterations. The eigenvectors of the diffusion tensor $\mathbf{D}$ are set equal to the eigenvectors $\mathbf{v}_{\mathbf{1}}, \mathbf{v}_{\mathbf{2}}, \mathbf{v}_{\mathbf{3}}$ with $\mathbf{v}_{\mathbf{1}}=\left[v_{11}, v_{12}, v_{13}\right]$ of the structure tensor (note the symmetry):

$$
\mathbf{D}=\left[\begin{array}{lll}
D_{11} & D_{12} & D_{13} \\
D_{12} & D_{22} & D_{23} \\
D_{13} & D_{23} & D_{33}
\end{array}\right] \quad \text { with } \quad D_{i j}=\sum_{n=1 . .3} \lambda_{n} v_{n i} v_{n j}
$$

The eigenvalues of the diffusion tensor are $\lambda_{1}, \lambda_{2}, \lambda_{3}$. Because our CBCT scans contain planar and tubular structures as well, we choose to use HDCS, with switches between CED and EED eigenvalues depending on the local image structure, for details see 6 . 
We can write the divergence operator equation 1 in $3 \mathrm{D}$ as:

$$
\nabla \cdot(\mathbf{D} \nabla u)=\partial_{x} j_{1}+\partial_{y} j_{2}+\partial_{z} j_{3}
$$

With $j_{1}, j_{2}, j_{3}$ the flux components which are described by:

$$
\begin{aligned}
& j_{1}=D_{11}\left(\partial_{x} u\right)+D_{12}\left(\partial_{y} u\right)+D_{13}\left(\partial_{z} u\right) \\
& j_{2}=D_{12}\left(\partial_{x} u\right)+D_{22}\left(\partial_{y} u\right)+D_{23}\left(\partial_{z} u\right) \\
& j_{3}=D_{13}\left(\partial_{x} u\right)+D_{23}\left(\partial_{y} u\right)+D_{33}\left(\partial_{z} u\right)
\end{aligned}
$$

For the standard discretization of the divergence operator central differences are used:

$$
\begin{aligned}
\partial_{y}\left(D_{12}\left(\partial_{x} u\right)\right)= & \frac{1}{2}\left(D_{12(i, j+1, k)} \frac{u_{(i+1, j+1, k)}-u_{(i-1, j+1, k)}}{2}\right. \\
& \left.-D_{12(i, j-1, k)} \frac{u_{(i+1, j-1, k)}-u_{(i-1, j-1, k)}}{2}\right)
\end{aligned}
$$

The other terms are written in the same way [8], and are combined to a pixellocation dependent $3 \times 3$ or $3 \times 3 \times 3$ convolution stencil. Non-negative discretization makes the modification that stencil elements remain positive for various gray values. Rotation invariant anisotropic diffusion is important with curved like structures such as the mandibular canal. Weickert [7] showed that larger stencils than $3 \times 3(2 \mathrm{D})$ are needed to fix the number of degrees of freedom to allow rotation invariance. This is achieved by implementing the equations 3 and 4, with Scharr's rotational invariant $3 \times 3$ filters for the image derivatives $\partial_{x}$ and $\partial_{y}$, resulting in an rotational invariant implicit $5 \times 5$ stencil.

\section{Optimized Scheme}

Another way to write the divergence operator using the product rule [9] is:

$$
\nabla \cdot(\mathbf{D} \nabla u)=\operatorname{div}(\mathbf{D}) \nabla u+\operatorname{trace}\left(\mathbf{D}\left(\nabla \nabla^{T} u\right)\right)
$$

We obtain for the divergence part of the equation:

$$
\begin{aligned}
\operatorname{div}(\mathbf{D}) \nabla u & =\left(\partial_{x} u\right)\left(\partial_{x} D_{11}+\partial_{y} D_{12}+\partial_{z} D_{13}\right) \\
& +\left(\partial_{y} u\right)\left(\partial_{x} D_{12}+\partial_{y} D_{22}+\partial_{z} D_{23}\right) \\
& +\left(\partial_{z} u\right)\left(\partial_{x} D_{13}+\partial_{y} D_{23}+\partial_{z} D_{33}\right)
\end{aligned}
$$

We write the Hessian part of the equation as:

$$
\begin{array}{r}
\operatorname{trace}\left(\mathbf{D}\left(\nabla \nabla^{T} u\right)\right)=\left(\partial_{x x} u\right) D_{11}+\left(\partial_{y y} u\right) D_{22}+\left(\partial_{z z} u\right) D_{33} \\
+2\left(\partial_{x y} u\right) D_{12}+2\left(\partial_{x z} u\right) D_{13}+2\left(\partial_{y z} u\right) D_{23}
\end{array}
$$

Equation 7 is discretized using $3 \times 3 \times 3$ derivative kernels, and the Hessian of equation 8 with a $5 \times 5 \times 5$ second derivative kernel. In $2 \mathrm{D}$ the spatial kernels can be written as: 


$$
\begin{aligned}
& M_{x x}=\left[\begin{array}{ccccc}
p_{1} & p_{2} & p_{3} & p_{2} & p_{1} \\
p_{4} & p_{5} & p_{6} & p_{5} & p_{4} \\
-p_{7} & -p_{8} & -p_{9} & -p_{8} & -p_{7} \\
p_{4} & p_{5} & p_{6} & p_{5} & p_{4} \\
p_{1} & p_{2} & p_{3} & p_{2} & p_{1}
\end{array}\right] \quad M_{x y}=\left[\begin{array}{ccccc}
p_{10} & p_{11} & 0 & -p_{11} & -p_{10} \\
p_{11} & p_{12} & 0 & -p_{12} & -p_{11} \\
0 & 0 & 0 & 0 & 0 \\
-p_{11} & -p_{12} & 0 & p_{12} & p_{11} \\
-p_{10} & -p_{11} & 0 & p_{11} & p_{10}
\end{array}\right] \\
& M_{x}=\left[\begin{array}{ccc}
p_{13} & p_{14} & p_{13} \\
0 & 0 & 0 \\
-p_{13} & -p_{14} & -p_{13}
\end{array}\right]
\end{aligned}
$$

The kernel values $\mathbf{p}=\left[p_{1}, p_{2} \ldots, p_{14}\right]$ can be found analytically or by numerical optimization. We choose numerical optimization, because it can optimize the whole process, while analytical derivation is only feasible for separate parts of the process, with simplifications such as ignoring numerical round of effects. We optimize the diffusion kernel using the following cost function:

$$
\mathbf{p}=\arg \min _{\mathbf{p}}\left(e_{f}(\mathbf{p})+\alpha e_{g}(\mathbf{p})\right)
$$

This function finds a balance between the edge orientation invariant filtering performance $e_{f}$, and isotropic diffusion performance $e_{g}$, with weight constant $\alpha$. With the first term $e_{f}$ we want to find the best edge enhancement for edges with several orientations and spatial frequencies. Therefore we use the difference between an image with circles of varying spatial frequencies without noise $I$, and an image with Gaussian noise added $I_{\text {noise }}$, which is diffusion filtered. With $F\left(I_{\text {noise }}, \mathbf{p}\right)$ the diffusion filtering of the image with noise using kernel values $\mathbf{p}$ :

$$
e_{f}(\mathbf{p})=\sum_{\mathbf{x}}\left|F\left(I_{\text {noise }}, \mathbf{p}\right)-I\right|, \text { with } I=\sin \left(x^{2}+y^{2}\right)
$$

With the second term $e_{g}$ we want to achieve Gaussian like diffusion in uniform regions. We use an image $I_{\text {point }}$ which is zero except the center pixel equal to one. The term $e_{g}$ is set to the difference between the isotropic noise filtered image $I_{\text {point }}$ and a least squares fitted Gaussian kernel. We set both diffusion tensor eigenvalues to one, corresponding to a uniform region.

$$
e_{g}(\mathbf{p})=\arg \min _{a} \sum_{\mathbf{x}}\left(F\left(I_{\text {point }}, \mathbf{p}\right)-\frac{1}{\pi \sqrt{a}} \exp \left(-|x|^{2} / a\right)\right)^{2}
$$

We use the Matlab Nelder-Mead Simplex minimizer [10] because it is robust against local minima. Also a quasi Newton minimizer is used [11, because the minimizer has a high convergence speed. We use 10 iterations of the Simplex Method followed by minimizing until convergence with the quasi Newton optimizer. This is done iteratively until the simplex method also converges. Parameters used for the circle image are, size $255 \times 255, \tau=0.1$, iterations $5, \sigma=1$, $\rho=10$, CED eigenvalues, Gaussian noise variance 0.1 , and $x$ and $y$ coordinates in the range $[-10,10]$. The parameters of $I_{\text {point }}$ are image size $51 \times 51$ and 5 iterations, constant $\alpha=200$. The computed kernel values $\mathbf{p}$ are: 


\subsection{0 .0490 .0320 .0380 .1110 .4480 .081 \\ 0.3340 .9370 .0010 .0280 .1940 .0060 .948}

It is important to note that the scheme is optimized for rotational invariance, but that the derivative kernels are not rotational invariant, for instance $M_{x}$ approximates a central difference instead of a Scharr like kernel.

In $3 \mathrm{D}$ the approach is the same, a spherical function in an image volume is used, with 33 instead of 14 unknown kernel variables. The optimized kernels are available in our open source diffusion toolbox 1 .

\section{Evaluation}

We evaluate the properties of the standard, rotation and optimized diffusion scheme with respect to three image based criteria. The first is noise removal in uniform regions, the second preservation and enhancement of image edges independent of rotation and size. The final test is the combined filtering performance on a real CBCT dataset.

In this first test we look at noise smoothing in uniform regions. To do this we use the image $I_{\text {point }}$ introduced in the optimization section, with the same filtering parameters and 100 iterations. Figure 1 shows the image results and difference between a least squares fitted Gaussian 2D function and the diffusion result. Ideal uniform diffusion is equal to Gaussian filtering, thus the standard diffusion and the optimized scheme perform well. The rotation invariant result does not look like a Gaussian, this is because the scheme is based on Sobel like derivative kernels, which do not use the local pixel value but only the neighboring values.

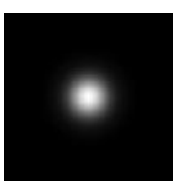

(a)

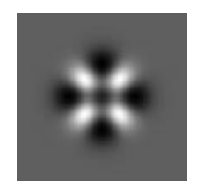

(b)

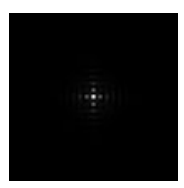

(c)

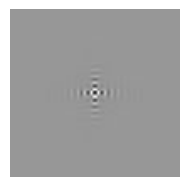

(d)

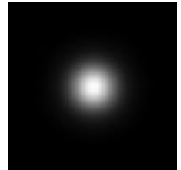

(e)

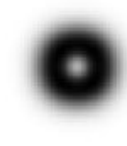

(f)

Fig. 1. Uniform Diffusion of a pixel with standard discretization $(a)$, rotation invariant $(c)$, optimized scheme $(e)$. Sub figures $(b),(d)$ and $(f)$ show the difference between the image result and least squares fitted $2 \mathrm{D}$ Gaussian function. The values in $(b)$ are in the order of $1 \cdot 10^{-5},(d)$ in the order of $1 \cdot 10^{-2}$ and $(f)$ in the order of $1 \cdot 10^{-4}$.

In the second test we look at rotation invariant edge enhancement, using the circle image with Gaussian noise $I_{\text {noise }}$, the same parameters as in the optimization section and 100 iterations.

Figure 2 shows that only the rotational invariant and optimized scheme are edge orientation independent. The rotational invariant scheme suffers from checkerboard artifacts due to the Scharr derivative kernels which only uses neighbor pixels and not the current pixel.

\footnotetext{
${ }^{1}$ Source code, http://www.mathworks.com/matlabcentral/fileexchange/25449
} 


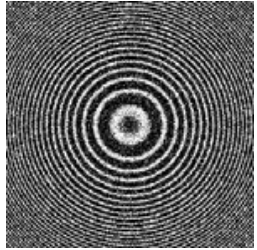

(a)

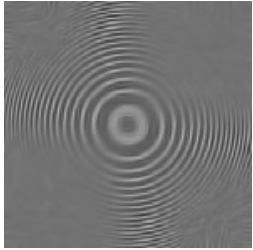

(b)

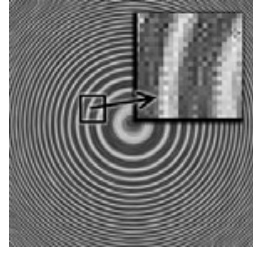

(c)

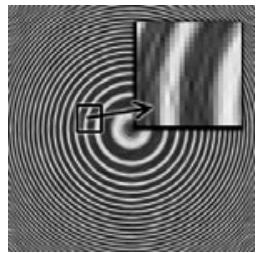

(d)

Fig. 2. The sub figures show the test image $(a)$, after diffusion with the standard scheme $(b)$, with the rotational invariant scheme $(c)$, and the optimized scheme $(d)$

The final test is performed on 8 CBCT preprocessed human-head datasets of $400 \times 400 \times 551$ voxels. The preprocessing consist of clustering the data sets in to three intensity classes background, tissue and bone, using bias field corrected fuzzy clustering [12, which is robust to streak artifacts. The resulting image data serves as ground truth for the edges. The edges are detected by applying a threshold on the gradient magnitude. Uniform regions are defined as the pixels which are at least six voxels away from an edge. Finally Gaussian noise of variance 0.01 is added to the image data. The image data is filtered with the standard and the optimized scheme using HDCS eigenvalues, with parameters $\sigma=0.5, \rho=2, \tau=0.15$, HDCS parameters $\lambda_{e}=30, \lambda_{h}=30, \lambda_{c}=15$ and 26 iterations, see figure 3 . Time to filter one dataset on an Intel Core 2 Duo desktop $\mathrm{PC}$ is approximately 25 minutes for the diffusion, and about 2.5 hours for the NLM filter.

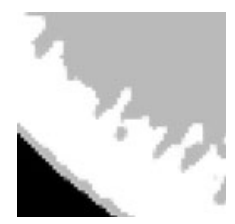

(a)

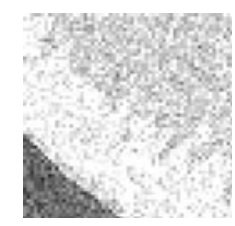

(b)

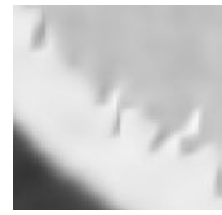

(c)

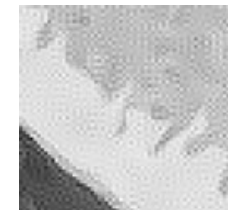

(d)

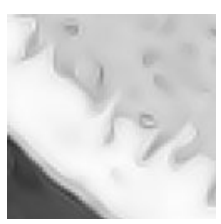

(e)

Fig. 3. Small part of HDCS filtered bone structure, ground truth (a), Gaussian noise added $(b)$, standard scheme $(c)$, rotation invariant $(d)$ and optimized scheme $(e)$

We compare the performance between the standard, the optimized diffusion scheme, and the original non-local means (NLM) [13]. The summed squared pixel distance between Gaussian low pass filtered and original diffusion results is used as a performance value. A steep edge contains high frequencies which will be removed by the low pass filter, resulting in a large pixel distance. In uniform regions high frequency noise will also be removed, thus a large pixel distance is a sign of noise which is not removed by the diffusion filtering. We calculate the smoothing pixel distance values for the edge pixels and for the uniform regions. The results are shown in table 1 . 
Table 1. Pixel distance between Gaussian smoothed and raw edge preserving diffusion filtering results of standard, optimized diffusion scheme and NLM

\begin{tabular}{ccccccccc}
\hline \multicolumn{4}{c}{ Edge } & \multicolumn{3}{c}{ Uniform region } \\
\hline dataset & raw & \multicolumn{2}{c}{ optimized standard } & NLM & raw & \multicolumn{2}{c}{ optimized standard NLM } \\
\hline 1 & $6.2 \cdot 10^{4}$ & $2.0 \cdot 10^{4}$ & $1.1 \cdot 10^{3}$ & $1.4 \cdot 10^{3}$ & $4.6 \cdot 10^{4}$ & $1.6 \cdot 10^{3}$ & 7.6 & 1.9 \\
2 & $6.6 \cdot 10^{4}$ & $2.1 \cdot 10^{4}$ & $1.5 \cdot 10^{3}$ & $2.1 \cdot 10^{3}$ & $4.7 \cdot 10^{4}$ & $1.2 \cdot 10^{3}$ & 6.7 & 1.5 \\
3 & $6.4 \cdot 10^{4}$ & $2.1 \cdot 10^{4}$ & $1.2 \cdot 10^{3}$ & $1.4 \cdot 10^{3}$ & $4.6 \cdot 10^{4}$ & $1.4 \cdot 10^{3}$ & 6.5 & 0.7 \\
4 & $6.5 \cdot 10^{4}$ & $2.0 \cdot 10^{4}$ & $1.5 \cdot 10^{3}$ & $2.2 \cdot 10^{3}$ & $4.7 \cdot 10^{4}$ & $1.2 \cdot 10^{3}$ & 6.2 & 3.1 \\
5 & $6.9 \cdot 10^{4}$ & $2.3 \cdot 10^{4}$ & $1.3 \cdot 10^{3}$ & $2.3 \cdot 10^{3}$ & $4.6 \cdot 10^{4}$ & $1.5 \cdot 10^{3}$ & 6.9 & 0.6 \\
6 & $6.7 \cdot 10^{4}$ & $2.6 \cdot 10^{4}$ & $2.0 \cdot 10^{3}$ & $1.6 \cdot 10^{3}$ & $4.5 \cdot 10^{4}$ & $2.2 \cdot 10^{3}$ & 9.9 & 0.3 \\
7 & $7.0 \cdot 10^{4}$ & $2.2 \cdot 10^{4}$ & $1.5 \cdot 10^{3}$ & $1.9 \cdot 10^{3}$ & $4.7 \cdot 10^{4}$ & $1.3 \cdot 10^{3}$ & 6.8 & 2.6 \\
8 & $6.4 \cdot 10^{4}$ & $2.2 \cdot 10^{4}$ & $1.3 \cdot 10^{3}$ & $1.8 \cdot 10^{3}$ & $4.6 \cdot 10^{4}$ & $1.4 \cdot 10^{3}$ & 6.7 & 1.6 \\
\hline
\end{tabular}

The NLM algorithm and standard scheme gives the best smoothing performance for uniform regions, with a 200 times smaller distance compared to the optimized scheme. This is because the optimized scheme preserved the edges of some random noise structures. The same noise structures are also visible in the rotation invariant scheme in image 3. In the HDCS eigenvalues there is a threshold value $\lambda_{h}$ to separate between noise and a image structures. But in this case the signal to noise ratio is too low to allow a good separation between noise and real image structures. Also on the real object edges the optimized scheme gives the highest pixel distance. This can be due to remaining noise on the edges or due to a steeper image edge than with standard scheme. Figure 3 shows it is because the image edges are more pronounced.

The original 8 CBCT datasets were also filtered with the three methods, and slices were shown to three medical experts which use cone-beam CT. They preferred the optimized filtering despited the fact it sometimes enhances noise structures. They explained that the other methods lose small important details, while the optimized filtering enhanced some hardly visible structures. Noise structures are not a major problem because the anatomy is known.

Finally we show the filtering results of all schemes on an CBCT scan which is geometric transformed to make the jaw flat, see figure 4 . The optimized scheme gives the best enhancement and preservation of the mandibular canal.

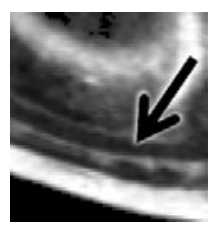

(a)



(b)

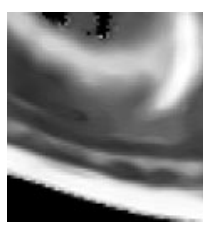

(c)

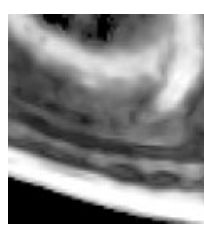

(d)

Fig. 4. Small part of HDCS filtered scan (a), mandibular canal (arrow), standard scheme $(b)$, rotation invariant $(c)$ and optimized scheme $(d)$. The optimized scheme better preserves the original edges and image structure. 


\section{Conclusion}

The introduced 2D/3D anisotropic diffusion scheme, shows better edge enhancement in our synthetic and CBCT data, compared to the standard, rotation invariant scheme and NLM. Filtering is Gaussian in uniform image regions without checkerboard artifacts. The results show that the better edge preservation also causes high noise structures to be preserved. Despite this artifact the medical experts preferred the introduced method because it enhanced also hardly visible anatomical structures. The cause of the problem is not the optimal scheme, but has to be solved by a better separation between noise edges and real edges in the diffusion tensor construction part.

\section{References}

1. Roberts, J., Drage, N., Davies, J., Thomas, D.: Effective dose from Cone-beam CT examinations in dentistry. Br. J. Radiol. 82, 35-40 (2009)

2. Sunnegardh, J.: Iterative Filtered Backprojection Methods for Helical Cone-Beam CT. PhD thesis, Computer Vision, The Institute of Technology (2009)

3. Awate, S., Whitaker, R.: Unsupervised, Information-Theoretic, Adaptive Image Filtering for Image Restoration. IEEE Trans. Pattern Anal. Mach. Intell. 28, 364376 (2006)

4. Perona, P., Malik, J.: Scale-space and Edge Detection using Anisotropic Diffusion. IEEE Trans. Pattern Anal. Mach. Intell. 12, 629-639 (1990)

5. Weickert, J.: Anisotropic Diffusion in Image Processing. PhD thesis, University of Copenhagen, Department of Computer Science (1998)

6. Mendrik, A., Vonken, E., Rutten, A., Viergever, M., van Ginneken, B.: Noise Reduction in Computed Tomography Scans using 3D Anisotropic Hybrid Diffusion with Continuous Switch. IEEE Trans. Med. Imaging 28(10), 1585-1594 (2009)

7. Weickert, J., Scharr, H.: A Scheme for Coherence-Enhancing Diffusion Filtering with Optimized Rotation Invariance. J. Vis. Commun. Image Represent. 13(1), 103-118 (2002)

8. Frangakis, A., Hegerl, R.: Noise Reduction in Electron Tomographic Reconstructions using Nonlinear Anisotropic Diffusion. J. Struct. Biol. (135), 239-250 (2001)

9. Felsberg, M.: On the Relation between Anisotropic Diffusion and Iterated Adaptive Filtering. In: Rigoll, G. (ed.) DAGM 2008. LNCS, vol. 5096, pp. 436-445. Springer, Heidelberg (2008)

10. Lagarias, J.C., Reeds, J.A., Wright, M.H., Wright, P.E.: Convergence Properties of the Nelder-Mead Simplex Method in Low Dimensions. SIAM J. Opt. 9(1), 112-147 (1998)

11. Shanno, D.: Conditioning of Quasi-Newton Methods for Function Minimization. Math. Comput. 24(111), 647-656 (1970)

12. Ahmed, M., Yamany, S., Farag, A., Moriarty, T.: Bias Field Estimation and Adaptive Segmentation of MRI Data Using a Modified Fuzzy C-Means Algorithm. In: Proc. IEEE Int. Conf. Comput. Vis. and Pattern Recognit., pp. 250-255 (1999)

13. Buades, A., Coll, B., Morel, J.: A Non-Local Algorithm for Image Denoising. In: Proc. of IEEE CVPR 2005, Washington, DC, USA, vol. 2, pp. 60-65. IEEE Computer Society, Los Alamitos (2005) 